\title{
Distribuição espectral e coeficientes de transmissão da radiação solar para condiçōes de céu limpo em Manaus
}

\author{
Ronaldo de Almeida $\left({ }^{*}\right)$ \\ Eneas Salati ( ${ }^{(*)}$ ) \\ Nilson Augusto Villa Nova ( ${ }^{(\vec{*} \#)}$
}

\begin{abstract}
Resumo
Medidas de radiação solar global e direta utilizando-se os filtros OG-530, RG-630 e RG-695 foram realizedas em Manaus-INPA. Os equipamentos disponiveis para estas medidas foram um piranômetro espectral $e$ um pireliômetro de incidência normal da Eppley. Os sinais foram registrados com o auxilio de um potenciógrafo LEEDS e NORTHRUP modelo SPEEDOMAX-W. As medidas foram realizadas em dois dias claros. Com os dados obtidos foi possivel estimar a variação horária do coeficiente de transmissão atmosférica correspondente a cada uma das faixas espectrais. Os autores apresentam uma distribuição horária dos fluxos de radiaçăo solar global direta e difusa.
\end{abstract}

INTRODUÇÃo

Com a crise mundial de energia gerada pelo alto custo do petróleo, foram intensificadas em todos os países, pesquisas sobre formas não convencionais de energia. Uma das alternativas para auxiliar na solução do problema energético é a utilização da radiação solar. No Brasil, programas específicos foram traça. dos em coordenação, especialmente através da FINEP (Financiadora de Estudos e Projetos). Nos estudos preliminares sobre a energia solar disponivel no Brasil, verificouse a escassez de dados sobre radiação solar, obtidos com instrumentos de precisão. Nos últimos cinco anos um esforço grande tem sido realizado para se estabelecer um mapeamento solarimétrico para as diversas regiões do País.

Para a região Amazônica alguns dados sobre radiação solar foram publicados recentemente. Villa Nova et al. (1976a) apresentaram estimativas do balanço de energia para diversos pontos da bacia Amazônica e a razão de insolação para 19 estações da região. Villa Nova et al. (1976b) apresentaram medidas do coeficiente de transmissão da radiação solar em Manaus no mês de junho. Villa Nova \& Salati (1977) apresentaram uma estimativa da radiação solar no Brasil, baseada na energia solar medida na passagem meridiana, das 97 estações estudadas, 15 pertencem a região Amazônica. Mota et al., 1977 apresentaram dados de radiação solar estimados para o Brasil e para a região Amazônica a partir das razões de insolação. Villa Nova et al. (1978) apresentaram dados medidos com precisão (utilizando pireliômetro Eppley) para radiação solar global na sede do INPA em Manaus.

Neste trabalho são apresentados os primeiros dados de radiação espectral obtidos na região Amazônica.

\section{MATERIAL E MÉTODOS}

\section{INSTRUMENTAL}

As medidas de radiação foram feitas em intervalos de 30 minutos na cidade de Manaus ( $3^{\circ} 08^{\prime} \mathrm{S}$ e $60^{\circ} 01^{\prime} \mathrm{W}$ ) nos dias $23-7-78$ (das $7: 00$ às $12: 00 \mathrm{~h}$ ) e 3-8-78 (das 12:00 às $17: 30 \mathrm{~h}$ ) quando o céu se apresentava isento de nuvens ou com poucas nuvens no horizonte.

A radiação global foi medida com um Eppley Precision Spectral Pyranometer acoplado a um registrador Speedomax-W, marca Leeds \& Northrup previamente calibrado.

Os valores espectrais de radiação global foram obtidos adaptando-se rápida e suces-

(*) - Universidade do Amazonas e Instituto Nacional de Pesquisas da Amazônia, Manaus.

(*) - Centro de Energia Nuclear na Agricultura-CNEN.SP e Instituto Nacional de Pesquisas da Amazônia, Manaus. (**) - Escola Superior de Agricultura "Luiz de Queiroz"-USP. 
sivamente sobre o radiômetro filtros de vidro (Scott glass filters) com as seguintes carac. terísticas :

Código antigo Código novo "Center of cut-off" Fator

\begin{tabular}{llll}
\hline OG 1 & OG 530 & 529 & 1.095 \\
RG 2 & RG 630 & 623 & 1.095 \\
RG 8 & RG 695 & 686 & 1.090 \\
\hline
\end{tabular}

A radiação incidente perpendicular à direção de propagação das ondas foi obtida com um Eppley Normal Incidente Pyrheliometer com os mesmos tipos de filtros já descritos, ligado a um milivoltímetro potenciométri. co, marca Leeds and Northrup, modelo 8690-2. sendo depois adequadamente convertida para unidades usuais de radiaçäo.

\section{EQUAÇÕES UTILIZADAS}

\section{Radiação direta}

Os valores medidos na direção dos raios solares foram multiplicados pelo cosseno do ângulo zenital no instante da observação, obtendo-se desta forma a radiação direta $(E)$, ou seja: a intensidade do fluxo de radiação solar que atinge diretamente uma superfície horizontal.

O cosseno do ângulo zenital foi calculado através da equação clássica (1)

$$
\begin{aligned}
\cos z= & \operatorname{sen} \phi \operatorname{sen} \delta+\cos \phi \cos \delta \cos h \ldots(1) \\
\text { onde }: z= & \text { ângulo zenital do sol no instante } \\
& \text { da observação } \\
\phi= & \text { latitude } \\
\delta= & \text { declinação do sol no instante da } \\
& \text { observação } \\
\mathrm{h}= & \text { ângulo horário do sol no instante } \\
& \text { da observação, determinado pela } \\
& \text { expressão: } \mathrm{h}=(12-\mathrm{H}) 1^{\circ}, \\
& \text { onde } \mathrm{H} \text { é a hora legal da observa. } \\
& \text { ção em horas e décimos. }
\end{aligned}
$$

\section{Radiação difusa}

A radiação difusa foi calculada subtraindo-se da radiação global a radiação direta.

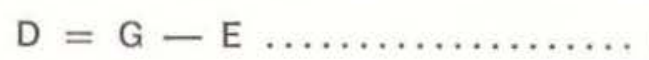

onde $: \mathrm{D}=$ radiação difusa

$\mathrm{G}=$ radiação global

$\mathrm{E}=$ radiação direta

\section{Radiação instantânea fora da atmosfera}

Os valores da radiação instantânea extraterrestre $\left(G_{\circ}\right)$ foram obtidos pela expressão

$$
\frac{\mathrm{dG}_{o}}{\mathrm{dt}}=\frac{\mathrm{J}_{a}}{\mathrm{R}^{2}} \cos \mathrm{z} \ldots \ldots \ldots \ldots \ldots \ldots
$$

onde : $\mathrm{R}=$ distância da Terra ao Sol expressa em termos do raio médio

$\mathrm{J}_{\mathrm{o}}=$ constante solar

\section{Coeficientes de transmissão}

Os coeficientes de transmissão da atmosfera para as faixas espectrais analisadas foram determinados através da equação (4)

$$
\mathrm{l}_{\mathrm{i}}=\mathrm{J}_{\mathrm{i}} 3 \sec \mathrm{z}
$$

onde: $\mathbf{Z}=$ coeficiente de transmissão da atmosfera

$I$ = radiaçäo incidente em uma superfície normal à direçäo dos raios solares ao nível do solo

$\mathrm{J}=$ radiação incidente em uma superfície normal à direção dos raios solares fora da atmosfera, integradas a partir da distribuição relatada por List (1966).

$z$ = ângulo zenital no instante da observação

$\mathrm{i}=$ faixa espectral considerada.

\section{Caracterização das faixas espectrais}

Segundo o fabricante, cada tipo de filtro utilizado apresenta uma transmissividade elevada a partir de um determinado comprimento de onda (center of cut-off), sendo pratica. mente opaco para comprimentos de onda menores que este valor. Desta forma, medindose um mesmo feixe de radiação com filtros 
QUADRO I Distribuição espectral da radiação solar (cal. $\mathrm{cm}^{-2} \mathrm{~min}^{-1}$ ) em Manaus (AM). Período da manhã (23/7/78). Período da tarde (3/8/78).

\begin{tabular}{|c|c|c|c|c|c|c|c|c|c|c|c|c|c|c|c|c|c|c|c|c|c|}
\hline \multicolumn{5}{|c|}{$\begin{array}{c}\text { total } \\
270-2500 \mathrm{~nm}\end{array}$} & \multicolumn{4}{|c|}{$\begin{array}{c}\text { violeta-azul } \\
270-529 \mathrm{~nm}\end{array}$} & \multicolumn{4}{|c|}{$\begin{array}{l}\text { verde-amarelo } \\
529-6623 \mathrm{~nm}\end{array}$} & \multicolumn{4}{|c|}{$\begin{array}{l}\text { vermelho } \\
623-686 \mathrm{~nm}\end{array}$} & \multicolumn{4}{|c|}{$\begin{array}{l}\text { infra-vermelho } \\
686-2500 \mathrm{~nm}\end{array}$} & \\
\hline Hora & G & $\mathrm{E}$ & D & 1 & $\mathrm{G}_{1}$ & $E_{1}$ & $D_{1}$ & $I_{1}$ & $\mathrm{G}_{2}$ & $\mathrm{E}_{2}$ & $\mathrm{D}_{2}$ & $\mathrm{I}_{2}$ & $\mathrm{G}_{3}$ & $E_{3}$ & $\mathrm{D}_{3}$ & $I_{3}$ & $\mathrm{G}_{4}$ & $E_{4}$ & $\mathrm{D}_{4}$ & $\mathrm{I}_{4}$ & \\
\hline $7: 00$ & 0,23 & 0,15 & 0,08 & 0,66 & 0,05 & 0,02 & 0,03 & 0,09 & 0,04 & 0,02 & 0,02 & 0,10 & 0,02 & 0,02 & 0,00 & 0,07 & 0,12 & 0,09 & 0.03 & 0,40 & \\
\hline $7: 30$ & 0,37 & 0,25 & 0.12 & 0.73 & 0,10 & 0,05 & 0,05 & 0,13 & 0.06 & 0,04 & 0,02 & 0,13 & 0,04 & 0,02 & 0,02 & 0.07 & 0,17 & 0,14 & 0,03 & 0,40 & \\
\hline $8: 00$ & 0,55 & 0,38 & 0,17 & 0.85 & 0,14 & 0,07 & 0,07 & 0,17 & 0.08 & 0,06 & 0,02 & 0,13 & 0,07 & 0,04 & 0,03 & 0,09 & 0,26 & 0,21 & 0,05 & 0.46 & \\
\hline $8: 30$ & 0,70 & 0,53 & 0.17 & 0,91 & 0,19 & 0,11 & 0,08 & 0,20 & 0,09 & 0,08 & 0,01 & 0,13 & 0,07 & 0,06 & 0,01 & 0,10 & 0,35 & 0,28 & 0,07 & 0,48 & \\
\hline 9:00 & 0,86 & 0,66 & 0,20 & 1.02 & 0,24 & 0,15 & 0,09 & 0,22 & 0,13 & 0,10 & 0.03 & 0.16 & 0,09 & 0,06 & 0,03 & 0.10 & 0,40 & 0,35 & 0,05 & 0,54 & \\
\hline $9: 30$ & 0,93 & 0,72 & 0,21 & 0,99 & 0,24 & 0,16 & 0,08 & 0.22 & 0,15 & 0,12 & 0,03 & 0,17 & 0,10 & 0,07 & 0,03 & 0,09 & 0,44 & 0,37 & 0.07 & 0,51 & \\
\hline $10: 00$ & 1,05 & 0,82 & 0,23 & 1,03 & 0,28 & 0,20 & 0.08 & 0,24 & 0.18 & 0,14 & 0,04 & 0,18 & 0,10 & 0,08 & 0,02 & 0,10 & 0,49 & 0,40 & 0,09 & 0,51 & \\
\hline $10: 30$ & 1,14 & 0,90 & 0,24 & 1,07 & 0,30 & 0,22 & 0.08 & 0,25 & 0.20 & 0,15 & 0.05 & 0.18 & 0,10 & 0,08 & 0,02 & 0,10 & 0,54 & 0,45 & 0,09 & 0,54 & \\
\hline $11: 00$ & 1,24 & 0,98 & 0,26 & 1.10 & 0,33 & 0,24 & 0,09 & 0,26 & 0,21 & 0.16 & 0.05 & 0.18 & 0,11 & 0,09 & 0.02 & 0,10 & 0,59 & 0,49 & 0,10 & 0.56 & \\
\hline $11: 30$ & 1,33 & 1,02 & 0,31 & 1,12 & 0,37 & 1,24 & 0,13 & 0,27 & 0,22 & 0,18 & 0.04 & 0,19 & 0,12 & 0,10 & 0,02 & 0.11 & 0,62 & 0,50 & 0,12 & 0.55 & \\
\hline $12: 00$ & 1,36 & 1.09 & 0.27 & 1.19 & 0,35 & 0,25 & 0,10 & 0.27 & 0,21 & 0,17 & 0.04 & 0.20 & 0,14 & 0,11 & 0,03 & 0.11 & 0,66 & 0,56 & 0,10 & 0,61 & \\
\hline Totais & 268,7 & 206,0 & 62.7 & 291,3 & 71,8 & 47.1 & 24,7 & 64,2 & 43,3 & 33,9 & 9,4 & 48,0 & 26,2 & 19,9 & 6.3 & 28,4 & 127,4 & 105,1 & 22,3 & 150,7 & \\
\hline $12: 00$ & 1,40 & 1,18 & 0,22 & 1,26 & 0,39 & 0,28 & 0,11 & 0,30 & 0,23 & 0,19 & 0,04 & 0,20 & 0,13 & 0,11 & 0,02 & 0.12 & 0,65 & 0,60 & 0,05 & 0,64 & \\
\hline $12: 30$ & 1,36 & 1,18 & 0,18 & 1,27 & 0,37 & 0,28 & 0.09 & 0,30 & 0,23 & 0.19 & 0,04 & 0,20 & 0,12 & 0,11 & 0,01 & 0,12 & 0,64 & 0,60 & 0,04 & 0,65 & \\
\hline $13: 00$ & 1,32 & 1,14 & 0.18 & 1.27 & 0,36 & 0,27 & 0,09 & 0.31 & 0,22 & 0,18 & 0.04 & 0,20 & 0,12 & 0.11 & 0,01 & 0,12 & 0,62 & 0,58 & 0.04 & 0.64 & \\
\hline $13: 30$ & 1,25 & 1,08 & 0,17 & 1,26 & 0,34 & 0,25 & 0,09 & 0,30 & 0,22 & 0,18 & 0.04 & 0.20 & 0.10 & 0.10 & 0,00 & 0,12 & 0,59 & 0,55 & 0.04 & 0,64 & \\
\hline $14: 00$ & 1,16 & 1,00 & 0.16 & 1,24 & 0,31 & 0,23 & 0.08 & 0,29 & 0.19 & 0,16 & 0.03 & 0.20 & 0,11 & 0.10 & 0,01 & 0.12 & 0,55 & 0,51 & 0,04 & 0.63 & \\
\hline $14: 30$ & 1,05 & 0,90 & 0,15 & 1,21 & 0,29 & 0,21 & 0.08 & 0,28 & 0,16 & 0,14 & 0,02 & 0,19 & 0,10 & 0,09 & 0,01 & 0,12 & 0,50 & 0,46 & 0.04 & 0,62 & \\
\hline $15: 00$ & 0,93 & 0,78 & 0.15 & 1,19 & 0,27 & 0,17 & 0,10 & 0,25 & 0,14 & 0,13 & 0,01 & 020 & 0,08 & 0,08 & 0,00 & 0.12 & 0,44 & 0,40 & 0,04 & 0,62 & \\
\hline $15: 30$ & 0.78 & 0,65 & 0,13 & 1,16 & 0,22 & 0,14 & 0.08 & 0,25 & 0,12 & 0,10 & 0,02 & 0,18 & 0,07 & 0,07 & 0.00 & 0,12 & 0,37 & 0,34 & 0.03 & 0.61 & \\
\hline $16: 00$ & 0,62 & 0,51 & 0,11 & 1.11 & 0,17 & 0,11 & 0.06 & 0,24 & 0,09 & 0,08 & 0,01 & 0,17 & 0,06 & 0,05 & 0,1 & 0,11 & 0,30 & 0,27 & 0,03 & 0.59 & \\
\hline $16: 30$ & 0,45 & 0,35 & 0,10 & 1,00 & 0,13 & 0,07 & 0,06 & 0,19 & 0,07 & 0,05 & 0,02 & 0.16 & 0,04 & 0,04 & 0.00 & 0.11 & 0,21 & 0,19 & 0,02 & 0.54 & \\
\hline $17: 00$ & 0,25 & 0,19 & 0,06 & 0,85 & 0,06 & 0,03 & 0.03 & 0,14 & 0,04 & 0,03 & 0,01 & 0,12 & 0,04 & 0,02 & 0,02 & 0,10 & 0,11 & 0,11 & 0.00 & 0,49 & \\
\hline $17: 30$ & 0,09 & 0.06 & 0.03 & 0.57 & 0,02 & 0.00 & 0,02 & 0,05 & 0,02 & 0,01 & 0,01 & 0,08 & 0,01 & 0,01 & 0.00 & 0.06 & 0,04 & 0,04 & 0.00 & 0,38 & \\
\hline$\left({ }^{\circ}\right)$ Totais & 296,1 & 251,2 & 44,9 & 367.5 & 81,5 & 57,0 & 24,5 & 80.9 & 48.1 & 40,0 & 8.1 & 57,8 & 26,8 & 24,8 & 2,0 & 368 & 139,7 & 129,4 & 10.3 & 192,0 & \\
\hline$(*)-$ Volo & lores intes & egrados pe & ela regra & a de Simp & & & & & & & & & & & & & & & & & 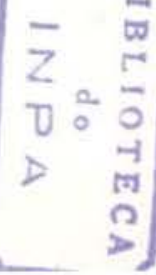 \\
\hline
\end{tabular}


de características cromáticas diferentes, pode-se determinar por subtração a intensidade da radiação na faixa espectral compreendida entre os mesmos.
Em face ao exposto, as seguintes combinaçöes de filtros nos permitiram detectar as diferentes faixas do espectro solar de acordo com o seguinte quadro :

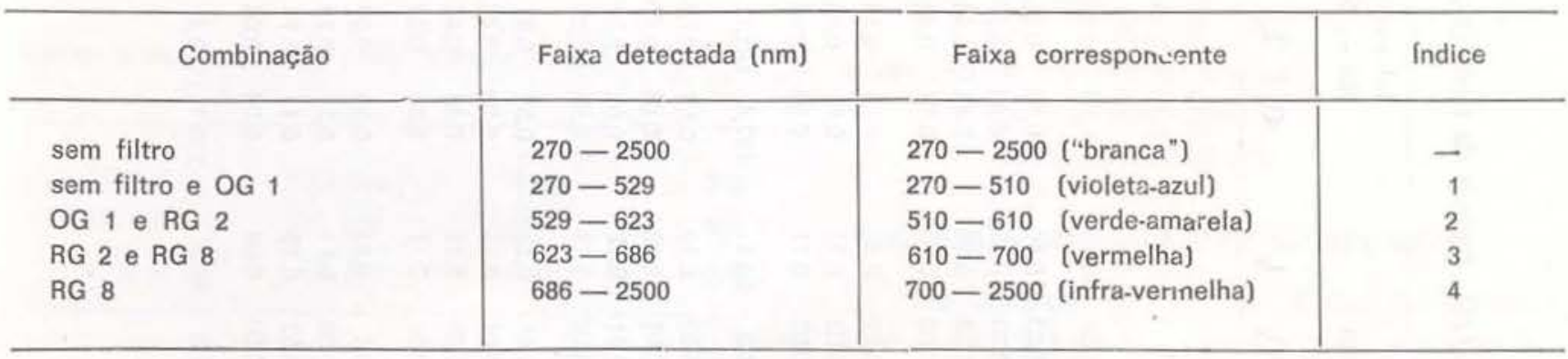

\section{RESULTADOS E DISCUSSÃO}

É muito difícil nesta época do ano ocorrer em Manaus um dia que se apresente totalmente sem nuvens, assim trabalhando no intervalo do dia 20-7-78 a 10-8-78 obteve-se uma manhã praticamente sem nuvens no dia 23-7 e uma tarde nas mesmas condições no dia 3-8.

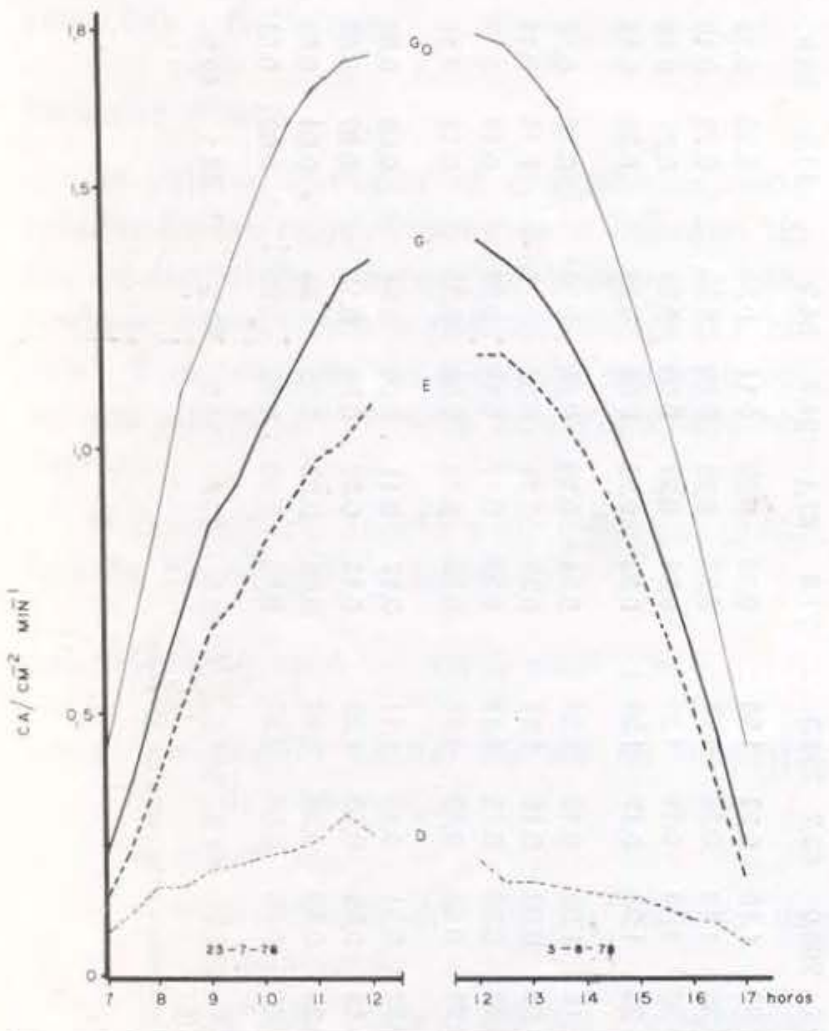

Fig. 1 - Distribuição horária da radiação extra-terrestre $\left(G_{0}\right)$, global $(G)$. direta $(E)$, e difusa (D). Período da manhã para o dia $23-7-78$ e o da tarde para 3-8-78. Manaus

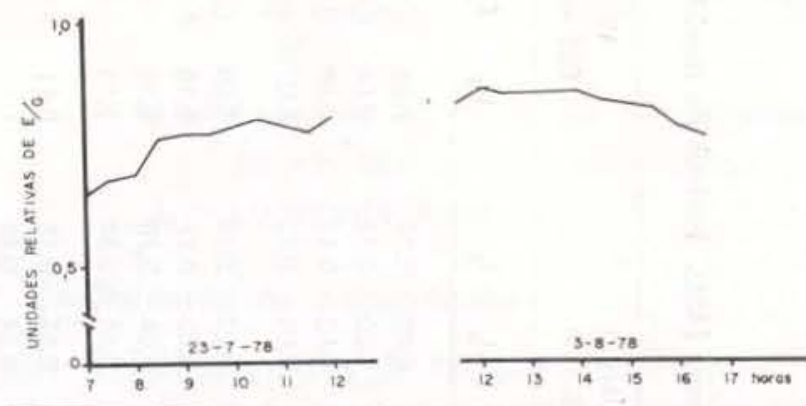

Fì. 2 - Variação horária da razão E/G

No quadro 1 estão indicados os valores instantâneos obtidos da radiação global (G), direta $(E)$, difusa (D) e recebida perpendicularmente aos raios (I) para a energia total do espectro solar, bem como os valores correspondentes para as diversas faixas espectrais analisadas.

A Figura 1 apresenta os valores de G, E e D. A curva envolvente $\left(G_{0}\right)$ indica a radiação incidente em uma superfície horizontal fora da atmosfera.

Os totais de radiação global recebidos nos períodos da manhã e tarde foram de $268,70 \mathrm{cal}^{\mathrm{cm}} \mathrm{cm}^{-2}$ e $296,10 \mathrm{cal} . \mathrm{cm}^{-2}$ respectivamente. Estima-se pois, que em um dia sem nuvens, nesta época do ano, devem chegar ao solo cerca de $570 \mathrm{cal} . \mathrm{cm}^{-2}$.

Na Figura 2 está indicada a relação horária entre a radiação direta e a global ao nível do solo. O valor médio obtido no período da manhã foi de $77 \%$ e no da tarde de $85 \%$. 

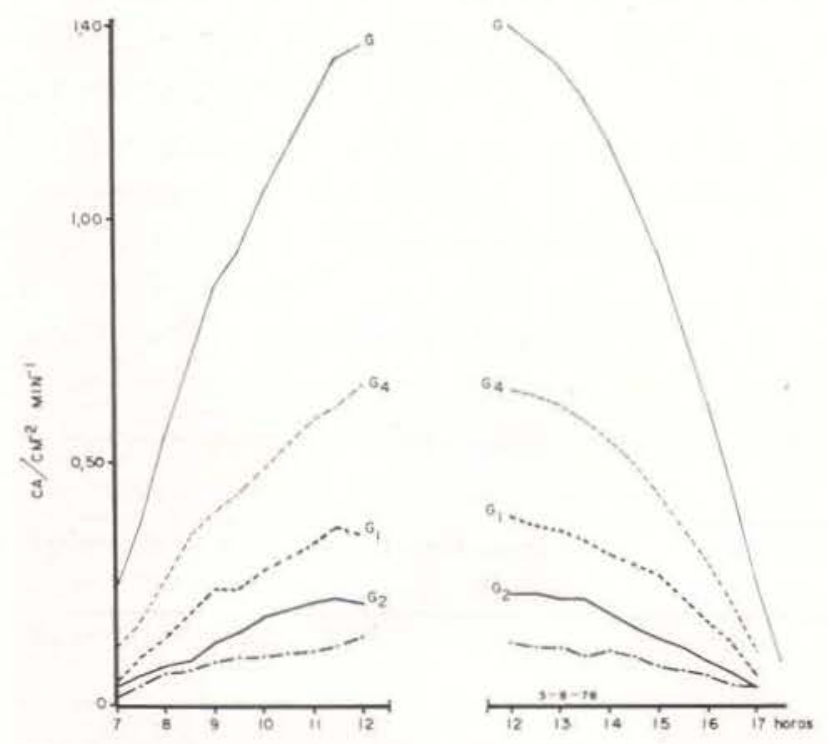

Fig. 3 - Distribuiçăo espectral da radiação global. G global total G 1 - violeta azul; G 2 - verde - emarelo; G 3 - vermelho; G 4 - infra - vermelho
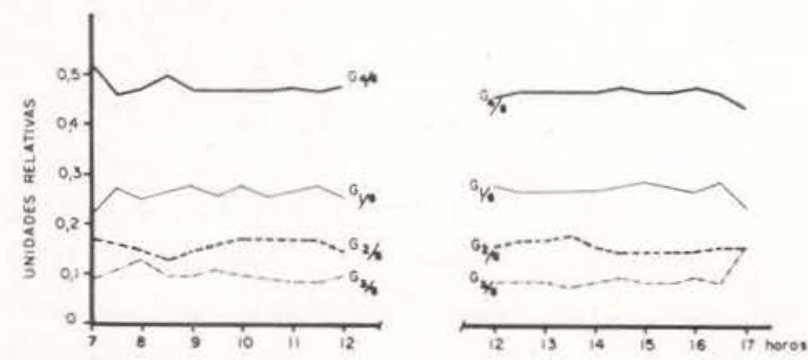

Fig. 4 - Relações entre as energias globais nas diversas faixas espectrais e a energia global total.

As curvas das Figuras 3 e 4 descrevem o comportamento da radiação global nas diversas faixas espectrais estudadas. $O$ infra-vermelho representa em média $47 \%$ da radiação global recebida tanto pela manhã quanto pela tarde. Com menores contribuições seguemse as regiōes do violeta-azul com $27 \%$, a do verde-amarelo com $16 \%$ e a do vermelho que participa com cerca de $10 \%$ do total. Nota-se também, que a composição espectral da radiação global se mantém praticamente constante durante todo o período.

A Figura 5 apresenta a radiação direta decomposta nas diversas faixas analisadas. A contribuição média de cada uma delas (Figura 6) segue a mesma ordem daquela apresentada para a radiação global, sendo $51 \%$ para o infra-vermelho, $23 \%$ para o violeta-azul, $16 \%$ para o verde-amarelo e $10 \%$ para o vermelho. A composição espectral da radiação direta está sensivelmente enriquecida pelo infra vermelho para ângulos zenitais aproximadamente maiores que $50^{\circ}$, (que corresponde aos períodos antes das 9:00h e depois das 15:00h).

A composição espectral da radiação difusa (Figura 7) apresenta-se formada, respectivamente nos períodos da manhã e da tarde, de $39 \%$ e $55 \%$ pela faixa violeta-azul, de $36 \%$ e $23 \%$ pela infra-vermelha, de $15 \%$ e $18 \%$ pela verde-amarela e de somente $10 \%$ e $4 \%$ pela faixa vermelha. Apesar dos periodos não serem de um mesmo dia, a sensivel
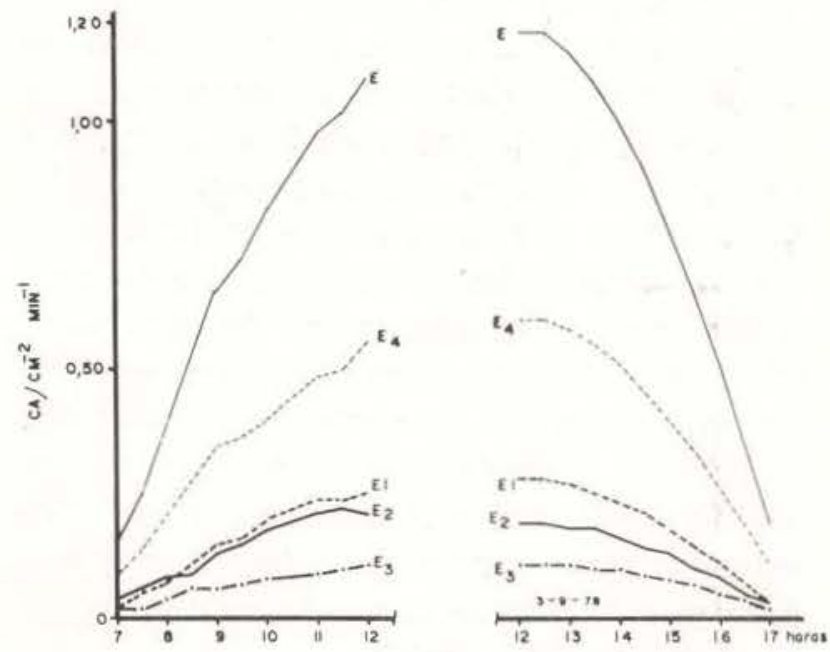

Fig. 5 - Distribuição espectral da radiaçăo direta : E direta total; E 1 - violeta-azul; E 2 - verde -amarela; E 3 - Vermelha; $E-4$ infra - vermelha.
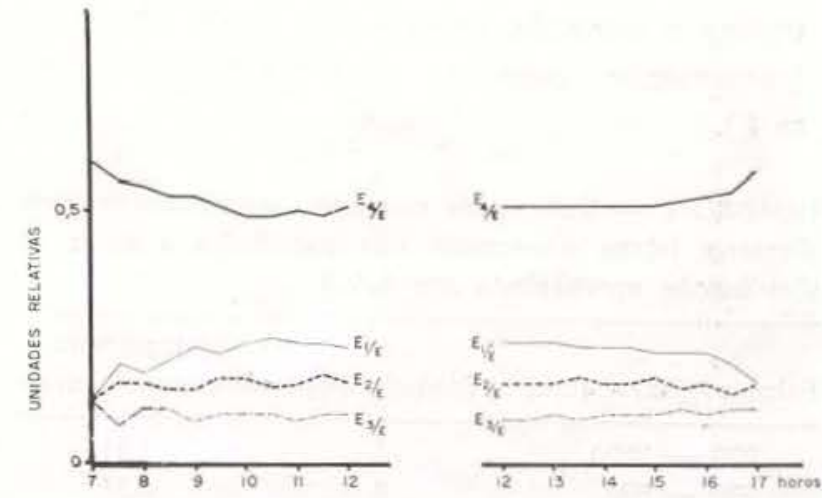

Fig. 6-Relaçōes entre as energias diretas nas diversas faixas espectrais e a energia direta total 

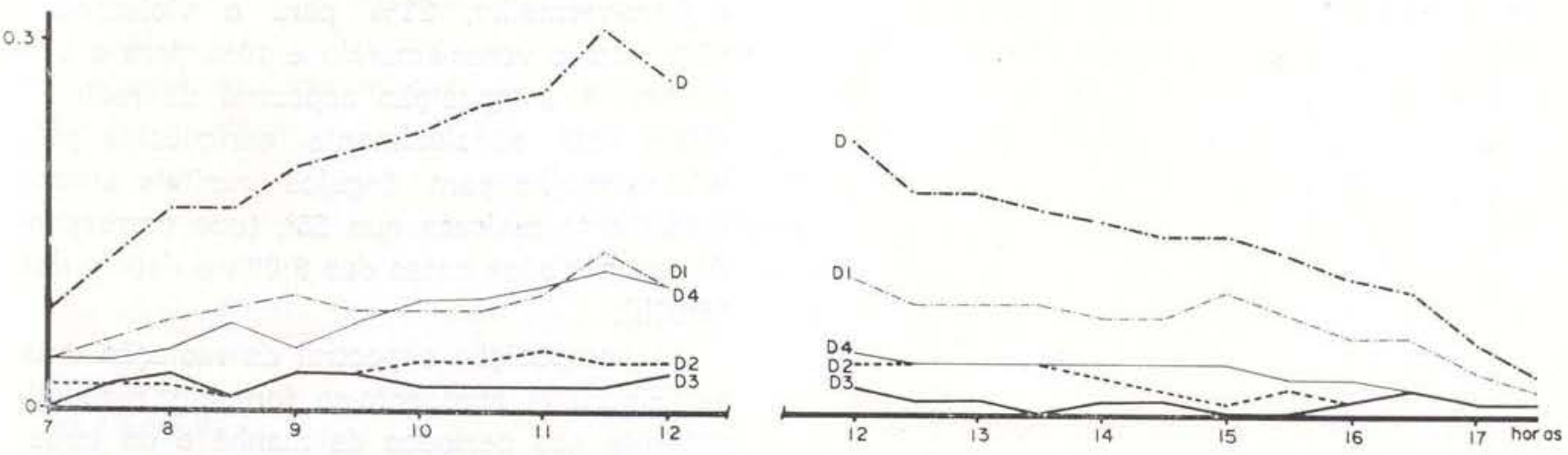

Fig. 7 - Distribuição espectral da radiação difusa. D - difusa total; D 1 - violeta - azul; D 2 - verde - amarela; D 3 - vermelha; D 4 - infra - vermelha

diferença entre os valores médios da parte da manhã e da tarde devem estar relacionados com as modificações da composição, da baixa atmosfera durante o transcorrer do dia.

A maior participação da faixa violeta-azul na radiação difusa, concorda com os dados da literatura, já que as moléculas formadoras do ar atmosférico interagem, preferencialmente, com os comprimentos de onda desta faixa intensificando, portanto, o seu espelhamento (scatering). Ademais, com o aumento do número de moléculas de água no ar, observa-se uma maior absorção da faixa infravermelha fazendo com que a importância desta faixa seja à tarde cerca da metade daquela do período da manhä.

O quadro 2 apresenta os valores de radiação extra-terrestre integrada nas diversas faixas espectrais analisadas $\left(\mathrm{J}_{\mathrm{i}}\right)$. Substituindo-se os valores de $J_{i}$ e $I_{i}$ na equação (4) obtivemos a variação horária dos coeficientes de transmissão para as diversas faixas (Figura 8).

QUADRO 2 - Valores da radiação extra-terrestre nâs diversas faixas espectrais $(\mathrm{Ji})$ calculados a partir da distribuição apresentada em S.M.T.

Intensidade

Faixa espectral $(\mathrm{nm})$ Símbolo $\left(\mathrm{J}_{\mathrm{i}}\right)$ cal. cm.-2 min-1

\begin{tabular}{lll}
\hline $270-2500$ & $\mathrm{~J}_{0}$ & 1,91 \\
$270-529$ & $\mathrm{~J}_{1}$ & 0,51 \\
$529-623$ & $\mathrm{~J}_{2}$ & 0,24 \\
$623-686$ & $\mathrm{~J}_{3}$ & 0,16 \\
$686-2500$ & $\mathrm{~J}_{4}$ & 0,99 \\
\hline
\end{tabular}

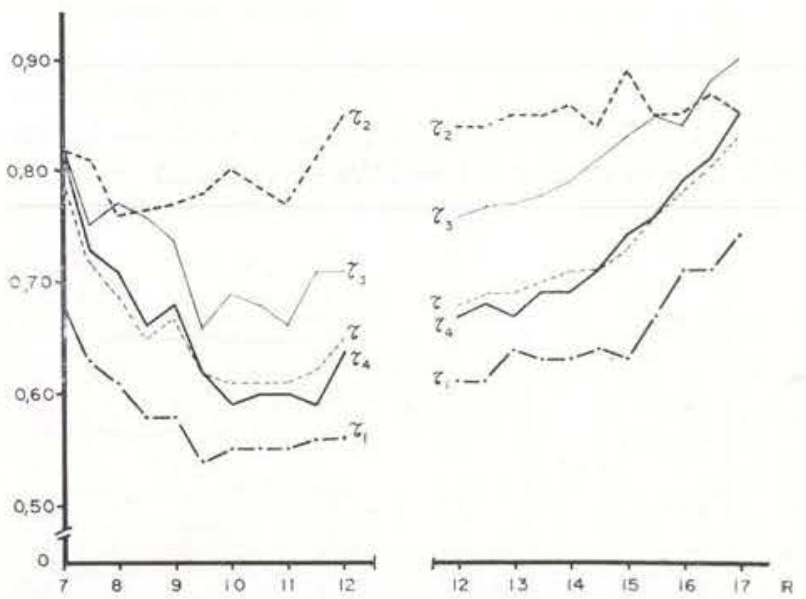

Fig. 8-Coeficientes de transmissão para diversas faixas espectrais: $1-$ total; $1_{1}-$ violeta - azul; $1_{2}-$ verde-amarela; $1_{3}-$ vermelha; $1_{4}-$ infra - vermelha.

\section{CONCLUSÕES}

A razão entre a radiação direta e a global foi em média $77 \%$ para o período da manhã e $85 \%$ para o período da tarde.

A composição espectral da radiação solar global mantém-se aproximadamente constante durante o periodo, sendo repartida em média $47 \%$ (infra-vermelho), $27 \%$ (violeta-azul), $16 \%$ (verde-amarelo) e 10\% (vermelho). Uma distribuição percentual praticamente idêntica foi observada para a radiação direta.

No que diz respeito à distribuição espectral da radiação difusa, observou-se uma variação acentuada entre os períodos da manhã 
e da tarde sendo 39 e $55 \%$ no violeta-azul, 36 e $23 \%$ no infra-vermelho e 15 e $18 \%$ na faixa verde-amarela.

Os valores médios dos coeficientes de transmissão estimados para os períodos da manhã e da tarde foram respectivamente 0,66 e 0,75 (radiação total), 0,58 e 0,67 (violetá azul), 0,78 e 0,86 (verde-amarelo), 0,66 e 0,75 para o infra-vermelho.

As determinações realizadas neste traba. Iho devem ser válidas para os dias limpos da estação seca, período no qual as características óticas das massas de ar predominante deveni ser idênticas.

\section{AGRADECIMENTOS}

Os autores agradecem a pesquisadora Maria de Nazaré Góes Ribeiro, chefe do Setor de Meteorologia do INPA, pelo apoio durante a execução do trabalho.

Este trabalho foi realizado com 0 apoio financeiro da FINEP (Projetos FINEP-USP IF/437 e FINEP $447 / \mathrm{CT}$ ) e da OEA (Projeto OEA-INPA : Utilização dos Recursos das Zonas Tropicais Úmidas - Ecologia da Floresta Tropical.

\section{SUMMARY}

Measurements of global and direct solar radiation utilizing OG-530, RG-630 and RG-695 filters were performed at INPA-Manaus. The equipment used ror these measurements was a set of Eppley Pyranometers. The signals were recorded with a Leeds and Northrup potentiographs. The measurements were performed during two clear days. With the dato obtained it was possible to estimate the hourly variation of the atmospheric transmission coefficients corresponding to each spectral band. An hourly distribution of the global, direct and diffuse fluxes of solar radiation is presented.

\section{BIBLIOGRAFIA}

LIST, R.J.

1966 - Smithsonian meteorological tables. 6 ed. Washington, Smithsonian Institution press. 527 p. Smithsonian Miscellanneous Collections, vol. 114

Mota, F.S.; BeISDoRf, M.I.C. \& AcostA, J.C.

1977 - Estimates of Solar Radiation in Brasil. Agricultural Meteorology, 18:241-254.

VIlla Nova, N.A. \& SAlatt, E.

1977 - Radiação Solar no Brasil. Anais do II Simpósio Anual da Academia de Ciências do Estado de São Paulo. Publicação ACIESP (6) : 27-61.

Villa Nova, N.A.; Salati, E. \& Matsut, E.

1976a - Estimativa da evapotranspiração da bacia Amazônica. Acta Amazonica, 6(2) : 215-228.

Villa Nova, N.A.; Salatt, E.; Santos, J.M. \& RIBEIRO, M.N.G.

1976b - Coeficientes de transmissẫo de radiação solar em Manaus em junho. Acta Amazo. nica, $6(3): 319-322$

Villa Nova, N.A.: Ribeiro, M.N.G.; Nobre, C.A. \& SALATI, E.

1978 - Radiação solar em Manaus. Acta Amazonica, $8(3): 417-421$.

(Aceito para publicação em 07/05/79) 